\title{
DERMATOFITOS EN POBLACION HUMANA Y ANIMAL EN ISLA DE PASCUA
}

\section{(Dermatophytes in human and animal population in Easter Island)}

\author{
*María Cristina Díaz J., ** Pablo Ostornol O, ***Ronald Diaz R \\ *Universidad de Chile ,Facultad de Medicina, ICBM, \\ Programa de Microbiología y Micología, Independencia 1027, Santiago, Chile. \\ ** alumno Escuela Tecnología Médica, \\ Universidad de Chile *** Laboratorio Central, Hospital Hanga Roa, Isla de Pascua
}

Palabras clave: Dermatofitos, antropofilicos, zoofilicos, Isla de Pascua Key words: Dermatophytes, antropophilic, zoophilic, Easter Island

\section{R ESUMEN}

Se determinó la presencia de dermatofitos en 85 muestras de raspado de piel de pacientes, $66 \mathrm{sin}$ lesiones aparentes y 19 con lesiones sugerentes de micosis, que consultaron al hospital Hanga Roa por causas no dermatológicas. Además se analizaron 86 muestras de animales (mediante la técnica del tapete), perros, gatos y equinos, sin lesiones aparentes de micosis. Las muestras clinicas fueron sembradas en Agar Sabouraud Glucosado y Lactritmel durante 21 días a 27 y $37^{\circ} \mathrm{C}$ y las de animales en DTM agar. Sólo 4 muestras de pacientes presentaron cultivos positivos (2 asintomáticos y 2 con lesiones). Los agentes aislados correspondieron a 1 M.canis, 2 M.gypseum y 1 T.rubrum. En los animales se obtuvieron 3 aislamientos de M. gypseum (sólo en perros y gatos).

Se evaluó la susceptibilidad "in vitro"de los agentes humanos aislados usando el método de microdilución en caldo, según recomendaciones NCCLS (documento M38-P), determinándose CIM para Fluconazol, Clotrimazol, Itraconazol, Griseofulvina y Terbinafina. Todos los antifúngicos evaluados resultaron ser activos frente a las cepas analizadas.

\section{INTRODUCCION}

La historia de la micología médica está estrechamente vinculada desde sus inicios con los dermatofitos y las dermatofitosis. Estos microorganismos son de distribución universal existiendo algunos endémicos en ciertas regiones geográficas. En Chile, las micosis superficiales no se incluyen en programas de salud pública ni constituyen enfermedades de notificación obligatoria, por lo que no es fácil tener una idea respecto de su

\section{ABSTRACT}

The presence of dermatophytes in 85 patients, 66 with no apparent lesions and 19 bearing possible mycosis lesions was confirmed after scraping their skin, even though they had attended the Hanga Roa hospital for causes other than dermatological. Besides, 86 samples from dogs, cats and horses, showing no apparent mycosis lesions were analysed (through the mat technique). Clinic samples were cultured in Glucose Sabouraud Agar and Lactrimel for 21 days at $27^{\circ}$ and $37^{\circ} \mathrm{C}$ and those from animals in DTM agar. Only 4 patient samples revealed positive cultures $(2$ asymptomatic and 2 with lesions). Isolated agents were identified as I M.canis, 2 M.gypseum and 1 T.rubrum. In animals 3 isolations of M.gypseum were achieved (only in dogs and cats).

The "in vitro" susceptibility of isolated human agents was evaluated by means of the broth microdilution method, as suggested by NCCLS (M38-P document) and CIM was selected for Fluconazole, Clotrimazole, Itraconazole, Griseofulvin and Terbinafine. All antifungal elements evaluated proved to be active in front of strains analysed.

prevalencia e incidencia, salvo por los diversos trabajos que se realizan en forma periódica en grupos seleccionados de población (Diaz, et al.,1990,2002; Piontelli et al. 1991; Zaror et al.,1991; Benavides et al., 1991)

La Isla de Pascua, cuya capital es Hanga-Roa, es el punto geográfico más aislado del mundo, ubicándose en el Océano Pacífico, a $27^{\circ} 08^{\prime}$ de latitud sur, y a $109^{\circ} 24^{\prime} 54^{\prime \prime}$ de longitud oeste, con una superficie de 179 $\mathrm{km}^{2}$, con costas abruptas y farellones rocosos. Su topografía es sinuosa, con cráteres volcánicos bien desarrollados, especialmente en sus vértices, lo que se 
traduce en una alta permeabilidad, con escurrimiento de las lluvias por lo que predomina una vegetación herbácea.

La posición de la Isla, permite que los vientos que sobre ella soplan sean muy húmedos, mientras que su situación subtropical le otorga temperaturas moderadas y homogéneamente altas, clasificándose su clima como, subtropical lluvioso; siendo la temperatura promedio de 20,4 $4^{\circ}$ (Cruz-Coke,1963). Estos antecedentes, permiten considerarla como un ecosistema insular particular que debido a sus condiciones climatológicas favorezca en cierta medida algunos tipos de afecciones dermatológicas sin dejar de considerar el origen étnico de su población con un importante componente polinésico (Englert, 1995). Los individuos relacionados con el cuidado y manejo de animales, están más expuestos a los dermatofitos geofilicos y zoofilicos, contribuyendo a la circulación y su distribución en la naturaleza de éstos u otros Onygenalés relacionados, además de otros microhongos anamorfos de Ascomycota potencialmente patógenos (Ajello \& Alpert, 1972; Piontelli \& Toro, 1987; Arenas, 2002).

Los estudios sobre este tema son escasos en la isla, en 1997 se analizaron 126 pacientes adultos residentes en Rapa Nui, obteniéndose un 5,5\% de prevalencia de dermatofitos, siendo $T$. mentagrophytes la especie más frecuente (Salamanca et al., 1997).

En los últimos años, se han introducido en la práctica clínica, nuevos antifúngicos, seguros y altamente efectivos, como son la Terbinafina y algunos azoles de última generación. Se ha observado en el tiempo que los dermatofitos han aumentado su resistencia a los antifúngicos (Norris et al., 1999). En base a lo expuesto y a la escasez de estudios dirigidos a la pesquisa de infecciones fúngicas en la población de esta isla, lo que impide conocer su epidemiología, el perfil de sensibilidad antifúngica y la real magnitud de las dermatofitosis.

El propósito de este estudio fue determinar la presencia de dermatofitos en población humana y animal, junto con evaluar su susceptibilidad antifúngica in vitro.

\section{MATERIALES Y METODOS}

Entre Enero y Marzo de 2003, se analizaron un total de 85 muestras clínicas, 66 de raspado de piel interortejo y plantar, de pacientes consultantes por causas no dermatológicas al Hospital Hanga Roa y 19 pacientes con lesiones en piel sospechosas de dermatofitosis. Las muestras se obtuvieron con un bisturí estéril, previa desinfección del área con alcohol $70 \%$, recolectándose en placas de Petri estériles y posteriormente cultivadas en Agar Sabouraud y Lactrimel, ambos adicionados de Cloramfenicol $(250 \mathrm{mg} / \mathrm{Lt})$ e incubados a $25^{\circ}$ y $37^{\circ}$ por un periodo entre 7 y 21 días. La identificación de los dermatofitos se basó en el manual de Hoog et al.(2000).

La pesquisa de dermatofitos como reservorio fúngico en perros, gatos y equinos se obtuvo según la técnica del tapete (Mariat \& Campos, 1967); los trozos de alfombra esterilizados en placas de Petri, permitieron escobillar el pelaje en varios sentidos sobre el cuerpo, patas, cuello y hocico del animal, para luego depositarlos nuevamente en sus respectivas placas y ser llevadas al laboratorio para su siembra mediante la impresión suave del tapete sobre toda la superficie de placas de Petri con Agar Dermatophyte Test Medium (DTM), siguiendo las mismas técnicas de incubación e identificación anteriormente mencionadas.

La susceptibilidad "in vitro" de los dermatofitos aislados en población humana se realizó usando el método de microdilución en caldo, según recomendaciones NCCLS (documento M38-P), determinandóse CIM para Fluconazol, Clotrimazol, Itraconazol, Griseofulvina y Terbinafina .

\section{RESULTADOS}

De las 85 muestras analizadas, 44 correspondieron al sexo femenino y 41 masculino, 66 de pacientes sin lesiones aparentes y 19 con lesiones sugerentes de micosis. Del total de muestras en 4 se obtuvo cultivos positivos $(4,7 \%), 2$ correspondieron a pacientes asintomáticos y 2 presentaron lesiones. Los agentes aislados correspondieron a 1 T.rubrum (hombre), 1 M.canis (mujer) y 2 M.gypseum (hombre y mujer). En 3/86 muestras de animales sin lesiones clínicas aparentes, se aislaron M. gypseum (2 gatos y un perro). Los dermatofitos aislados de población humana presentaron los siguientes rangos de : Fluconazol $0.13-32 \mu \mathrm{m} / \mathrm{mL}$, Itraconazol 0.03 0.25 , Clotrimazol 0.03-0.06, Griseofulvina 0.03-0.06 y Terbinafina 0.03. (Tabla. 1)

\section{DISCUSION}

Los ecosistemas insulares presentan generalmente características ecológicas particulares en relación a la presencia o ausencia del hombre y su flora y fauna. En la Isla de Pascua, en especial, la densidad poblacional ha ido en aumento en los últimos años por razones turísticas. Ajello \& Alpert (1972), analizando 44 muestras de suelos en esta Isla encontrando una variedad de hongos queratinofilicos entre los que se destacan 22 aislamientos del complejo Microsporum fulvum-gypseum.(44.9\%) y 1 (2.1\%) de Trichophyton ajelloi. A diferencia de un estudio realizado en suelos de Papua (Nueva Guinea), en que no se aislaron dermatofitos antropofílicos y zoofílicos pero si Keratinomyces ajelloi como 
CIM de dermatofitos aislados en humanos frente a los antifúngicos evaluados

\begin{tabular}{|l|c|c|c|c|}
\hline \multirow{2}{*}{ ANTIFÚNGICOS } & $\begin{array}{c}\text { M. canis } \\
\text { * CIM ug/mL }\end{array}$ & $\begin{array}{c}\text { M. gypseum 1 } \\
\text { CIM ug/mL }\end{array}$ & $\begin{array}{c}\text { M. gypsezan 2 } \\
\text { CIM ug/mL }\end{array}$ & $\begin{array}{c}\text { T. nubrim 1 } \\
\text { CIM ug/mL }\end{array}$ \\
\hline Fluconazol & 32 & 1.0 & 0.13 & 0.5 \\
\hline Itraconazol & 0.25 & 0.06 & 0.13 & 0.03 \\
\hline Clotrimazol & 0.03 & 0.03 & 0.06 & 0.03 \\
\hline Griseofulvina & 0.03 & 0.06 & 0.06 & 0.03 \\
\hline Terbinafina & 0.03 & 0.03 & 0.03 & 0.03 \\
\hline
\end{tabular}

*CIM : concentración que inhibe el $80 \%$ de los microorganismos.

colonizadores de sustratos queratínicos (FilipelloMarchisio et al., 1991). Por otro lado, también se reporta que en los suelos de Malasia de 98 hongos queratinofilicos geofilicos aislados 34 fueron $\boldsymbol{M}$.gypseum. (SooHoo,1991). A pesar que en nuestro estudio no se incluyó el muestreo de suelos, llama la atención que en los animales solo se aisló M.gypseum. Este aislamiento podria explicarse por el contacto directo constante de los animales domésticos con la tierra. M. gypseum se aisló de gatos y un perro, coincidiendo en cierta medida con lo reportado por Efuntoye \& Fashanu (2002), donde los agentes más frecuentes en los animales estudiados correspondieron a $\boldsymbol{M}$. canis y $\boldsymbol{M}$. gypseum. Nakamura et al. (1999), demostraron que existía un fuerte incremento en el número de casos de dermatofitosis humanas transmitidas desde animales.

Comparado con Chile continental, al parecer en la isla se encuentra una diferente distribución de dermatofitos presentes en la población humana y animal, esto podría explicarse por las costumbres de la población que habitualmente camina con pies descalzos y a no utilizan zapatos cerrados debido al calor. Por otro lado no existe un alto hacinamiento y se utilizan raramente los baños públicos.

A pesar de la distribución cosmopolita de los dermatofitos, los estudios realizados en islas o en zonas geográficas aisladas en el mundo son escasos. En nuestro análisis global se determinó un $4.7 \%$ de aislamiento de estos agentes, lo que concuerda con un estudio anterior realizado en 126 isleños sanos y con lesiones en que la prevalencia de dermatofitos fue de $5.5 \%$ (T . mentagrophytes en 6 casos y T. rubrum en uno, Salamanca et al., 1997). A pesar que no es posible comparar otras islas del mundo de mayor extensión, población y condiciones raciales mixtas, se aprecia claramente una diversa distribución, con un aparente claro predominio de dermatofitos antropofílicos, seguido por los zoofílicos. (Suarez et al., 1994 en Cuba; Maraki \& Tselentis, 1998 en Creta; Ng et al., 2002 en Malasia; Piqué et al., 2002 en Lanzarote) coincidiendo generalmente con los resultados obtenidos en los continentes. Por el contrario, llama la atención que en la Isla de Pascua parece haber un claro predominio de los zoofilicos y geofilicos sobre los antropofilicos A pesar que los geofilicos integrantes del complex M.gypseum-fulvum se consideran como raros patógenos oportunistas en el hombre, puede ser que su alta distribución(44,9\%) en los suelos de la isla (Ajello \& Alpert,1972) como los (35\%)encontrados en Malasia (Soo-Hoo,1991), sean cepas adaptadas para crecer a temperaturas más altas cercanas a los $37^{\circ} \mathrm{C}$ y por ende pasar directamente del suelo a la piel humana (especialmente los pies) o al sobrevivir en el pelaje de los animales junto a su microbiota normal ya sea como saprófitos o patógenos débiles y ser trasmitidos al hombre por el contacto directo o indirecto con su pelaje. Llama la atención la ausencia de $\boldsymbol{T}$. mentagrophytes en estos últimos aislamientos, la especie dominante en el estudio de Salamanca et al. (1997) y la ausencia del complex $\boldsymbol{M}$. gypseum-fulvum.

Los dermatofitos aislados fueron sensibles frente a los antifúngicos probados, excepto en una cepa de $\boldsymbol{M}$. canis que presentó una CIM de $32 \mathrm{mg} / \mathrm{L}$ para Fluconazol, clasificado como sensible, dosis dependiente probablemente porque es el único disponible en la farmacia, estos resultados son similares a los obtenidos por otros autores (Jessup et al., 2000; Ghannoum et al., 2000; Díaz et al., 2002).

Resulta dificil realizar estudios epidemiológicos en una etnia con una cultura, idiosincracia, idioma y costumbres totalmente disímiles y más complicado aún si se trata de un investigador chileno continental, ya que este pueblo ha sido objeto de minuciosos estudios de antropólogos, etnólogos, genetistas, médicos, etc.

La periodista Malú Sierra (2002), los define de la siguiente manera: "Desconfiados como todos los isleños, pero doblemente desconfiados por ser infinitamente isleños, los rapanui miden y pesan al forastero en una sola mirada. Están aburridos de ser observados como si fueran insectos y no ganar nada a cambio ".

En conclusión, podemos comentar que la presencia de dermatofitos es baja, que los agentes son semejantes a los reportados en el continente, pero varía su prevalencia y distribución, siendo $\boldsymbol{T}$. rubrum el agente aparentemente menos aislado en la población humana. 
La sensibilidad antifúngica de estos queratinofilicos parece no diferir de lo encontrado en el continente. Se requieren mayores investigaciones para corroborar si con el continuo aumento del cambiante flujo poblacional, la distribución y la sensibilidad pueda variar en el tiempo.

\section{REFERENCIAS}

Ajello, L. \& Alpert, E .(1972) .Survey of eeaster island soils for keratinophylic fungi Mycologia 64:161-166

Arenas, R. (2002). Dermatofitosis en Mëxico Rev .Iberoam. Micol. 19: 63-67

Benavides, M.I.; Mondaca, X.; Olate, C.; Vogel, M.; Rodriguez, B.(1991). Diagnóstico de laboratorio de las dermatofitosis: experiencia de 10 años en el área occidente de Santiago. Rev.Méd.Chile 119: 1029- 1032

Cruz-Coke, R. (1963) .Ecología Humana de la Isla de Pascua. Rev. Med.Chile 91:73-79

De Hoog, G.S.; Guarro,J. ; Gené, J. \& Figueras, M.J. (2000). Atlas of clinical fungi. 2nd Ed.CBS, Neder., Univ. Rovira i Virgili, Reus, Spain

Díaz, M.C.; Fich ,F.\& Salamanca, L. (1990).Agentes Etiológicos de Micosis superficiales en un área de Santiago - Chile ( 1977- 1987). Boletín Micológico $5: 5-8$

Diaz, M.C.; Roessler, P.; Fich, F.; Gómez, O.; Ostornol, P.; Pérez, L. (2002). Dermatofitosis: Etiologia y susceptibilidad antifúngica in vitro en 3 centros hospitalarios de Chile. Boletin Micológico, Chile 17:101107

Efuntoge, M.O.\& Fashanu, S.O. (2002) Fungi isolated from skins and pens of helthy animals in Nigeria. Mycophatologia; 153:21-23

Englert, S. (1995).La tierra de Hotu Motua: Historias y Etnología de Isla de Pascua. Santiago, Editorial Universitaria.pp. 41-58

Filipello,Marchisio, V.; Curetti, D.; Cassinelli, C. ; Bordese, C . (1991) Keratinolytic and keratinophilic fungi in the soils of Papua New Guinea. Mycopathologia 115: 113-119

Ghannoum M.A, Hajjeh R.A, Scher R, Konnikov N, Gupta A.K, Summerbell R, Sullivan S, (2000).A large-scale North American study of fungal isolates from nails : the frecuency of onicomicosis, fungal distribution and antifungal susceptibility patterns J. Am. Acad. Dermatol. 43:641-648

Jessup,C.J.;Warner, J.;Isham, N.; Hasan, I .; Ghannoum, M.A .(2000) Antifungal susceptibility testing of dermatophytes: Establishing a medium for inducing conidial growth and evaluation of susceptibility of clinical isolates. Journal of Clinical Microbiology 38: 341-344
Maraki, S. \& Tselentis ,Y.(1998). Dermatophytoses in Crete, Greece, between 1992 and 1996. Mycoses 41:175-178

Mariat, F. \& Campos, A.C. (1967). La technique du carré de tapis, methode simple de prelevament dans les micoses superficielles. Ann. Inst. Pasteur 113:666

Nakamura ,Y.; Watanabe, S. \& Hasegawa, A. (1999) Dermatomycosis in human and animals. Nipon Ishinfin Gakkai Zasshi 40:9-14

National Committe for Clinical Laboratory Standard. (1997). Reference method for broth dilution antifungal susceptibility testing of yeasts. Approved Standard M 27. National Committe for Clinical Laboratory Standards, Villanova, Pensylvania.

National Committe for Clinical Laboratory Standard.(1998). Reference method for broth dilution antifungal susceptibility testing of conidiumforming filamentous fungi; Proposed Standard M 38-P. National Committe for Clinical Laboratory Standards, Villanova, Pensylvania.

Ng ,K.P.; Soo-Hoo, T.S.; Na, S.L.; Ang, L.S. (2002).Dermatophytes isolated from patients in University Hospital, Kuala Lumpur, Malaysia. Mycopathologia.; 155:203-6

Norris, H. A.; Elweski, B.E. \& Ghannoun, M.A. (1999). Optimal growth conditions for the determination of the antifungal susceptibility of three species of dermatophytes with the use of a microdilution method, J. Am. Acad. Dermatol. 40:9-13

Piontelli ,E.\& Toro M.A.(1987). Los animales domésticos (perros y gatos) como reservorio fúngico. Boletín Micológico 4:149-158

Piontelli. E.; Toro, M. A \& Jara, D. (1991). Micosis superficiales en pacientes de servicios dermatológicos de la $\mathrm{V}$ región : estudio de prevalencia en el periodo 1984-1989. Boletin Micológico 6: 63-68

Piqué E, Fusté R, Copado R, Noguera J, Ramis P ( 2002). Estudio de las dermatofitosis en Lanzarote ( 1995-1999) Rev Iberoam Micol 19: 165 168

Salamanca, L.; Díaz, M.C.; Yazigi, R.; Ovalle C.(1997). Presencia de Dermatofitos en adultos de Isla de Pascua. III Congreso de Infectología del Cono Sur, XIV Congreso Chileno de Infectologia. Valdivia.

Sierra, M.(2000). Rapa Nui . Naufragos del planeta 1ra Edición, Editorial Persona.

Soo Hoo, T.S. (1991).Isolation of keratinophilic fungi from soils in Malasya Mycopathologia 113: 155-158

Suarez, M.; Estrada, A.; Peláez, R.; Pérez, Y.; Perez, J.; Rosell, P. (1994). Dermatofitosis en la Provincia de Ciego de Ávila..Cuba. Boletín Micológico 9: $121-123$

Zaror, L.; Lorca, S.\& Martinez, C. ( 1991 ). Dermatofitos en reclutas y áreas de riesgo en regimientos de la ciudad de Valdivia ( Chile ). Boletin Micológico $6: 27-31$ 